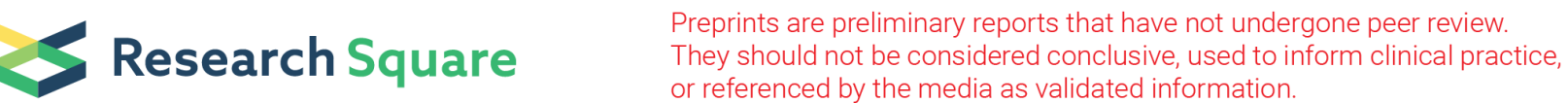

\section{Why Don't Japanese Early Childhood Educators Intervene in Children's Physical Fights? Some Characteristics of the Mimamoru Approach}

Fuminori Nakatsubo ( $\nabla$ nakatsub@hiroshima-u.ac.jp )

Hiroshima University https://orcid.org/0000-0002-0086-0962

Harutomo Ueda

Nagoya City University https://orcid.org/0000-0001-8175-7731

Misa Kayama

University of Mississippi https://orcid.org/0000-0002-7276-0733

\section{Research Article}

Keywords: Japanese early childhood education and care, Japanese early childhood educators, U.S. early childhood educators, Mimamoru approach, Children's physical fights

Posted Date: April 6th, 2021

DOI: https://doi.org/10.21203/rs.3.rs-356531/v1

License: (9) This work is licensed under a Creative Commons Attribution 4.0 International License.

Read Full License

Version of Record: A version of this preprint was published at Early Childhood Education Journal on April 12th, 2021. See the published version at https://doi.org/10.1007/s10643-021-01184-3. 


\section{Abstract}

This study aims to examine pedagogical meanings of the Mimamoru approach frequently used by Japanese early childhood educators in children's physical fights. Mimamoru is a strategy in which educators intentionally withhold an intervention, while carefully observing children, to foster children's voluntary participation in their own learning, socially and cognitively. In this report, we examine why Japanese educators tend not to intervene, and how they determine whether their intervention is necessary. Using methods from Tobin's video cued multi-vocal ethnography, we conducted focus groups at 9 early childhood education and care facilities (7 in Japan and 2 in the U.S.) with a total of 34 Japanese and 12 U.S. educators. They watched a short video clip in which a mid-career male teacher, one of the Japanese participants, used the Mimamoru approach with two children involved in a physical fight. Educators, then, discussed their interpretations of the teacher's responses to children. The analyses of participants' discussion suggest that educators' non-intervention, an important feature of the Mimamoru approach, provides children with opportunities to autonomously learn interpersonal skills, for example, through the experiences of feeling guilty and solving problems by themselves. Yet educators do intervene when they determine that the risk of physical harm caused by fighting is greater than the benefit for children to learn. Implications of the Mimamoru approach, including in other cultural contexts, are discussed.

\section{Introduction}

Early childhood education plays important roles in supporting children's development across cultures (e.g., The World Bank, 2017). In Japan, 97\% of children ages 3 to 6 attend early childhood education and care (ECEC) facilities (Ministry of Education, Culture, Sports, Science, and Technology, 2019). A primary goal of Japanese ECEC is to nurture and cultivate children's attitude to, and motivation for, acquiring knowledge; and promote their development (e.g., in motor, critical thinking, and decision making skills) through children's voluntary play within peer groups (Ministry of Education, Culture, Sports, Science and Technology, 2017). Thus, many Japanese ECEC facilities have little emphasis on children's acquisition of academic skills. Likewise, Japanese early childhood educators understand that children's cognitive, social and emotional skills can be better developed within everyday-life contexts, particularly during play, through their regular interactions with others, rather than adults' direct instruction and guidance using top-down approaches (e.g., Doi et al., 2005; Ministry of Education, Culture, Sports, Science and Technology, 2018).

Japanese ECEC, especially educators' indirect guidance for children to learn appropriate behaviors, frequently through their experiences of handling conflicts and frustration within peer groups, has drawn the attention of scholars from Western countries (e.g., Lewis, 1995; Walsh, 2004). A premise of this strategy is the educators' use of implicit, indirect strategies that minimize their direct guidance and intervention, such as staying back intentionally from children facing problems (Lewis, 1995; Tobin et al., $1989,2009)$. Japanese early childhood educators across various ECEC facilities, for example, promote children's social and emotional development by creating a social context in which children play together 
freely, solve problems with peers, and learn social skills. In doing so, educators may make a variety of play equipment available, rather than finding faults in every single act of children and warning them (Walsh, 2004). These implicit strategies may reflect Japanese child rearing and socialization practices, such as mimamoru, that aim to promote children's internal motivation to learn through their everyday social interactions, in contrast to the use of external reinforcement, such as praising and punishment (e.g., Doi et al., 2005; Levine \& White, 2003.)

\section{Mimamoru as a Japanese social and cultural practice}

Mimamoru (mi: watch; mamoru: guard/protect) is a Japanese pedagogical strategy in which adults, including early childhood educators (Tobin et al., 2009), intentionally withhold an intervention to promote children's learning through their voluntary exploration and actions (e.g., Bamba \& Haight, 2011).

Mimamoru generally is understood as "teaching by watching"; that is, a so-called hands-off or nonintervention approach that indirectly fosters children's cognitive and social development (Hayashi, 2011; Tobin \& Hayashi, 2015). The Mimamoru approach, however, is not written in the Japanese national ECEC curriculum standard (Burke \& Duncan, 2014; see also Ministry of Education, Culture, Sports, Science and Technology, 2017). It rather is considered an implicit curriculum, which generally reflects culturally based socialization and educational practices (Wang et al., 2008). In Japanese socialization practices at home and school, for instance, adults frequently wait for children to respond to problems and guide them to take ownership of their own learning (Author 3, 2020). Although such Mimamoru approach looks passive, it rather challenges educators to remain patient, watching and waiting for children to think and act on their own (Author 3, 2014; Tobin et al., 2009). An underlying assumption of this Japanese practice is adults' trust in children's inherent goodness, more specifically, their ability to learn through everyday social interactions (e.g., Doi et al., 2005; Lewis, 1995; Walsh, 2004). Yet "watching" does not mean that adults ignore the safety of children. They do intervene if they determine that children need adults' direct guidance. In other words, children learn through their exploration of autonomy under the protection of adults (Bamba \& Haight, 2011).

The Mimamoru approach is widely used in various fields in Japan, such as special education (Author 3, 2014, 2020) and child welfare (Bamba \& Haight, 2011), as well as ECEC. Educators' mimamori (noun form of mimamoru) of elementary school-aged children with disabilities, for example, allowed children to learn and negotiate meanings of their differences from typically-developing peers by creating a safe environment in which children can experience minor conflicts with peers, such as teasing, and recover from them (Author 3, 2014, 2020). At a child welfare institution in Japan, staff members' mimamori also created socially and emotionally supportive contexts in which children with maltreatment histories felt safe, developed relationships with supportive adults, and overcame challenges (Bamba \& Haight, 2011).

\section{Mimamoru approach in children's physical fights}

How adults handle children's physical fights varies across cultures (Author 3, 2014; Tobin et al, 2009). The examination of Japanese educators' strategies for intervening in physical fights, in contrast to those from other cultures, underscores unique characteristics of the Mimamoru approach. For example, U.S. early 
childhood educators, relative to Japanese educators, tend to immediately intervene and correct children's inappropriate behaviors, particularly fights (Tobin, et al., 1989; 2009). Although educators may tolerate physical fights of young children, for example, as rough play, unlike older children (e.g., Carlson, 2011), they consider incidents involving aggressive behaviors seriously. U.S. early childhood educators, indeed, frequently intervene in such behaviors by taking children's privileges away, sending them to time-out, and at times removing them from the classroom (e.g., Paley, 2004; Tobin et al., 2009), including out-of-school suspensions (e.g., Bayat, 2019). Thus, early childhood educators are responsible for stopping children's aggressive behaviors and guiding them to learn appropriate social and problem solving skills.

In contrast, Japanese early childhood educators often use the Mimamoru approach, and encourage children involved in physical fights to make their own decisions and solve conflicts (Hayashi \& Tobin, 2015). In Japan, children's behaviors such as hitting peers are not necessarily considered to require adults' immediate intervention. These behaviors are, rather, viewed as social immaturity. Children, for instance, may become frustrated when they are unable to verbalize their feelings, and engage in physical fights (e.g., Kubo, 2011). Physical fights, thus, are understood as important opportunities to teach children appropriate emotional and social skills, and promote their character development that is necessary to become responsible adults (Peak, 1991; Tobin, et al., 2009).

Japanese early childhood educators' preference of not intervening immediately in children's physical fights is considerably different from educators in other countries, particularly, those from Western countries (e.g., Author 3, 2014; Fujita \& Sano, 1988; Tobin, et al., 2009). Similar to the U.S., for example, the national curriculum of New Zealand (Te Whariki) describes early childhood educators' responsibility for preventing children's fights, for instance, by preparing a sufficient number of play equipment and materials (Burke \& Duncan, 2014). Japanese educators, however, may deliberately prepare less than enough amount of materials and equipment for play to intentionally create a situation in which children experience conflicts and learn how to share limited materials available (Burke \& Duncan, 2014; see also Hayashi \& Tobin, 2015). Still, when children begin to fight, Japanese educators prepare to intervene, including by moving closer to children and observing them carefully to determine if any intervention is necessary (Hayashi \& Tobin, 2015). In other words, educators allow children to solve disputes by themselves while adjusting their location and distance from them, so that they can intervene when necessary. This indirect intervention is considered a central feature of the Mimamoru approach.

\section{The current study}

This study aims to understand; 1) why Japanese early childhood educators tend not to intervene, and 2) how and in what contexts they, then, intervene, through the examination of the Mimamoru approach utilized by Japanese early childhood educators in children's physical fights. Currently, the majority of studies on the Mimamoru approach at Japanese ECEC facilities have been conducted by Tobin and Hayashi (Hayashi 2011; Hayashi et al., 2009; Hayashi \& Tobin, 2011, 2012, 2015; Tobin \& Hayashi 2015; Tobin et al., 1989; 2009). Their studies examine characteristics of the Mimamoru approach, such as Japanese early childhood educators' non-verbal, implicit behaviors, for example, the appropriate distance 
from children that is "close enough to make a suggestion, but far enough away to suggest [and intervene]" (Hayashi \& Tobin, 2015, p.30). We, however, examine the Mimamoru approach from the pedagogical perspective. Our aim is to understand educational meanings of educators' non-intervention strategies, for example, what they expect to occur by not intervening immediately, and how they balance the risk of potential harm as a result of non-intervention with any benefit for children. The examination of the pedagogical meanings of non-intervention strategies will provide a new perspective on behavior interventions and disciplinary practices, particularly for educators from countries in which direct behavior interventions are prioritized.

[1] For example, preschools typically serve children ages 3-6, with a stronger focus on "education", relative to day care centers serving children of all ages before starting $1^{\text {st }}$ grade.

\section{Methodology}

\section{Research design: Video cued multi-vocal ethnography}

This study used methods from video cued multi-vocal ethnography (see Tobin, 1989; Tobin et al, 1989; 2009), with some modifications. Tobin and his colleagues, originally, used 20 minute video clips of a "Typical Day" at several preschools taken and edited by researchers to facilitate focus groups in which educators from multiple cultures discussed their interpretations of culturally nuanced socialization practices with children, including the Mimamoru approach. In this study, however, we used a shorter, 3 minute video clip that captured the Mimamoru approach utilized to handle a physical fight between two children. Our methods, still, have benefited from the use of video cued approach that allows for the discussion of non-verbal behaviors of children and educators, such as the timing of intervention, which may be difficult to ask using semi-structured interview guides or scenarios.

Similar to Tobin's approach, data were collected through focus groups. Japanese and U.S. early childhood educators discussed the Mimamoru approach utilized by one Japanese teacher in response to two boys involved in a physical fight at our primary research site, Sakura Preschool (pseudonym), a private preschool, located in a rural, forest area in Western Japan. Sakura preschool is known for its unique programs based on the Mimamoru approach, in which children work in the nearby forest to learn various skills, such as outdoor cooking, guided by educators who utilize indirect, non-intervention strategies that value children's autonomy in their learning.

\section{Video clip on children's fight}

We used a short video clip (approximately 3 minutes) on a physical fight between two three-year-old boys (Ken and Takuya[2]) from a documentary film entitled "Naite Waratte [Crying and Smiling]," broadcasted as a TV program by Nippon Housou Kyoukai (NHK; Japan Broadcasting Corporation, 2012, May 18). This 40 minute documentary film followed children at Sakura Preschool for over one year. After watching the documentary film, we contacted an administrator of the preschool and obtained permission to conduct a focus group with educators on several scenes from the film. We have followed the guideline determined 
by the NHK (2021) to use the documentary film in this research project, including the use of photos (Figures 1-4) in publications.

This report focuses on four consecutive scenes (Figures 1-4) of the physical fight between Ken and Takuya. [Insert Figure 1 around here.] One morning, Ken and Takuya are arguing over a butterfly net. Ken refuses to share the butterfly net with Takuya, who is crying. Their argument evolves into a physical fight. They hit each other initially. Takuya, then, bites on Ken's arm. Kumi, a five-year-old girl who is a leader of this multi-age group, is observing the situation. Mr. Yoshida, a mid-career teacher who is present at the scene, says nothing, but puts his hand on Ken's arm and tries to stop Takuya's biting (Figure 1). He, however, does not stop their physical fight. Rather, Mr. Yoshida quietly steps back and watches the children from a position some distance away from them (Figure 2). [Insert Figures 2 and 3 about here.] Other children watching Takuya and Ken fighting also begin to leave for the playground. Kumi, however, stays with them, and steps in to help them make up (Figure 3):

Kumi [to Takuya]: Hey, say you are sorry. What's wrong?

Takuya: Because Ken didn't let me use [the butterfly net].

Kumi: ...But Takuya, you bit him, right? So, you must apologize. Got it? Ken also is bad because he didn't lend you the net, but you bit him, right? So both of you should apologize. OK?.... Then say, "I'm sorry."

Takuya: I'm sorry.

Kumi: Ken must say, "Sorry," too.

Ken: I'm sorry.

Mr. Yoshida and other teachers briefly looked at Kumi, Takuya, and Ken while playing with other children in the playground. After apologizing to each other, the three children walk to the playground (Figure 4). [Insert Figure 4 about here] Teachers and other children accept them without saying anything, and they begin to play together.

The use of this video clip allowed us to facilitate a focused discussion with early childhood educators on the use of Mimamoru approach in children's physical fights. Indeed, Mr. Yoshida's responses in these scenes provide an important case example of the Mimamoru approach, for instance, his adjustment of the distance from Ken and Takuya experiencing emotional reactions (see Hayashi and Tobin, 2015). During focus groups, participants elaborated on their interpretations of such non-verbal, as well as verbal responses of children and educators, including meanings of the distance between Mr. Yoshida and children.

Further, the video clip, taken by professional documentarists with clear image and sound, stimulated participant educators' reflection on the Mimamoru approach. This documentary film has drawn the attention of a number of early childhood educators across Japan for the intensive, and successful use of 
the Mimamoru approach at our primary research site. Note that the audio of this video clip is in Japanese, and we have added captions in English for U.S. participants.

\section{Participants}

Along with our primary site, Sakura Preschool, six other ECEC facilities in Japan located in middle-sized western cities and two preschools in the U.S., one in midwestern, and another in northeastern cities, were selected as our secondary sites, from where we recruited our participants. These sites were selected purposively to include both public and private ECEC facilities. We expect that perceptions of educators from Japanese private ECEC facilities are similar to those of our primary site. Educators at Japanese public ECEC facilities that are required to follow the National Curriculum Standard for Early Childhood Education can diversify our interpretation of the Mimamoru practice at our primary site. These secondary sites are considered typical ECEC facilities in both Japan and the U.S. The size of our research sites varies from approximately 20 , at the primary site, to 120 .

We recruited participants from both primary and secondary sites to address variations in their perceptions within and across cultures. First, the teacher shown in the video clip, Mr. Yoshida, and his colleagues at Sakura Preschool can provide insider interpretations of their Mimamoru approach. Second, Japanese early childhood educators from other ECEC facilities may address variations in interpretations among educators across Japan. Finally, U.S. early childhood educators can provide a cross-cultural view of how educators handle children's physical fights. Their cross-cultural view allows us to identify characteristics of the Mimamoru approach Japanese educators take for granted as self-evident, and further explore their cultural meanings (see also Author 3, 2020).

A total of 46 educators from our primary (4 educators, including Mr. Yoshida) and secondary sites (30 Japanese and 12 U.S. educators) participated in this study. In order to obtain diverse perspectives, we recruited 30 teachers and 4 administrators in Japan, and 10 teachers and 2 administrators in the U.S. All educators we have contacted agreed to participate in focus groups (see Table 1).

\section{Table 1}

Participants, research sites, and timeline 


\begin{tabular}{|c|c|c|c|c|}
\hline $\begin{array}{l}\text { Research } \\
\text { sites }\end{array}$ & $\begin{array}{l}\text { Type of ECEC } \\
\text { facilities }\end{array}$ & $\begin{array}{l}\text { Number of participants } \\
\text { (Female / Male) }\end{array}$ & $\begin{array}{l}\text { Average years of } \\
\text { experiences }\end{array}$ & $\begin{array}{l}\text { Date of } \\
\text { inquiry }\end{array}$ \\
\hline \multicolumn{5}{|c|}{$\begin{array}{l}\text { Primary site, } \\
\text { Japan }\end{array}$} \\
\hline $\begin{array}{l}\text { Sakura } \\
\text { Preschool }\end{array}$ & Private & $4(3 / 1)$ & 5.8 & Oct. 2013 \\
\hline Total & & $4(3 / 1)$ & & \\
\hline \multicolumn{5}{|c|}{ Secondary sites, Japan } \\
\hline JA & Private & $3(2 / 1)$ & 25.0 & Feb. 2015 \\
\hline$J B$ & Private & $4(4 / 0)$ & 12.0 & Mar. 2020 \\
\hline $\mathrm{JC}$ & Public & $4(3 / 1)$ & 19.0 & Mar. 2020 \\
\hline$J D$ & Public & $7(6 / 1)$ & 21.0 & Mar. 2020 \\
\hline JE & Public & $6(5 / 1)$ & 12.7 & Mar. 2020 \\
\hline JF & Private & $6(5 / 1)$ & 9.5 & Mar. 2020 \\
\hline Total & & $30(25 / 5)$ & & \\
\hline \multicolumn{5}{|c|}{ Secondary sites, U.S. } \\
\hline UA & Private & $6(5 / 1)$ & 9.2 & Mar. 2013 \\
\hline UB & Public & $6(5 / 1)$ & 19.2 & Jan. 2013 \\
\hline Total & & $12(10 / 2)$ & & \\
\hline
\end{tabular}

\section{Procedures}

This study has been approved by [the institution Author 1 is affiliated with], and conducted, following the research ethics guidebook published by the Japan Society of Research on Early Childhood Care and Education (2012).

We collected data over 8 years, from 2013 to 2020 . We contacted Sakura Preschool in 2013, immediately after the documentary film was broadcasted, to obtain feedback from educators who worked directly with children featured in the film. We also have conducted follow up discussions with educators at Sakura Preschool in 2020 to make certain that our data are comparable to those collected at Japanese secondary sites in 2020 . U.S. data were collected in 2013 , in order to compare with our initial findings at Sakura Preschool.

At each ECEC facility, an audio-recorded focus group was conducted. At the beginning of the focus groups, researchers provided a brief description of the documentary film, showed the video clip involving the four scenes of children's fight, and asked participants to freely discuss the scenes. Researchers asked 
probe questions as necessary to facilitate participants' discussions. During the focus groups, all but three participants discussed the four scenes for about 15-40 minutes.

\section{Data analysis}

All recorded focus group interviews were transcribed verbatim in the original languages. The transcripts in English, then were translated into Japanese by a research member fluent in both English and Japanese. All analyses were conducted in Japanese, a common language of the research team. Only illustrative excerpts were translated into English, except for quotes from transcripts in English. In order to understand embedded meanings in the participants' narratives, we analyzed the data guided by the procedure developed by Otani (2019). More specifically, after repeated, and thorough reading of the transcripts, we identified noteworthy words and phrases (e.g., those that were recurrent or culturally nuanced) that could be used as "clues" to understand the Mimamoru approach, such as "minimal intervention", "supporting children's autonomy" and "problem solving". Using these words and phrases as codes, we then conducted line-by-line coding, with careful consideration of the meanings of participants' narratives. A total of 118 codes were generated. Using all of these codes, we reconstructed a storyline that highlighted underlying meanings of the Mimamoru approach utilized by Mr. Yoshida through discussion within our research team. The storyline was critiqued by 3 research members and revised until all members agreed. Findings of this study have been presented at the primary site for the purpose of member check (Lincoln \& Guba, 1985).

[2] All names are pseudonyms, different from names used in the original documentary film.

\section{Results}

Japanese and U.S. early childhood educators' responses to the video clip illuminated three major characteristics of the Mimamoru approach. First, Mr. Yoshida's "temporary, minimal intervention" reduced the immediate risk of physical harm. Next, "non-intervention" in which Mr. Yoshida stepped back and watched the children fighting from a distance indirectly encouraged them to solve their own problem. This strategy also guided them to learn appropriate behaviors by experiencing the consequence of physical fight. Finally, "non-presence" in which he stayed further away allowed an older child to step in and helped the two children make up.

\section{Temporary, minimal intervention}

When Ken and Takuya began physically fighting, Mr. Yoshida's initial response was touching Ken's arm to stop Takuya's potentially harmful behavior, biting (see Figure 1). Mr. Yoshida's response in this scene can be characterized as a temporary, minimal intervention, which eliminates an immediate risk.

\section{Judging whether intervention is necessary}

During the focus group at Sakura Preschool, Mr. Yoshida described his interpretation of Takuya's and Ken's behaviors, "I put my hand on Ken's arm to prevent bite marks on his arm. Hitting each other with 
bare hands can be tolerated, though." This was the only intervention Mr. Yoshida employed in this video clip. Another teacher from Sakura Preschool provided a similar, but slightly different perception, "[There is no need to stop the fighting.] Also, I wouldn't intervene at all. Takuya isn't seriously biting Ken's arm. He's going easy." Mr. Yoshida agreed, "Sure. Maybe I could have waited a little longer." They understand that biting is acceptable unless it is serious, and stopping their physical fights is not necessary.

Yet, of 30 Japanese educators from the secondary sites, all 27 educators who commented on this scene described that they would intervene earlier than Mr. Yoshida and his colleagues at Sakura Preschool, and might stop either hitting or biting. One teacher, for example, stated,

If I were in the position of Mr. Yoshida, I would just tell them, 'Don't hit!' as long as they are hitting with bare hands, but I would not step in [and stop them] when they just are hitting. I would stop them when biting begins.

This teacher considers that children's hitting behavior is caused by their frustration, especially when they are not able to verbalize their feelings. Hitting is also considered physically less harmful than biting. Thus, the necessary intervention in this scene, for her, was to stop the biting. Another teacher interpreted somewhat differently, "I would intervene when they begin hitting with bare hands." She understood that children's hitting, generally, was a violent attempt to harm their friends, and stopping them would be considered a necessary intervention. In response to these educators, Mr. Yoshida and his colleagues at Sakura Preschool described that this strategy, providing a minimal intervention was based on their discussion, and agreement with children's parents. Without parents' approval, they would have to implement more direct interventions, just like other Japanese educators described.

U.S. educators also elaborated on concerns about children's safety. Their interpretations reflect U.S. educational practices and contexts. Children's physical attack is understood as requiring educators' immediate intervention to protect children from abuse and violence, following federal and local child protection[3] and school disciplinary policies (e.g., U.S. Department of Education, 2017). One U.S. teacher, for instance, described their responsibility, "Certainly, it does bring up concerns about regulation and policy, ...having to talk to parents about why their child has a bite ring on their forearm, but on the other hand, they [behaviors] ended pretty quickly." Another U.S. teacher also described, "As soon as there was physical harm, we would step in, immediately." Holding back, or keeping their intervention at a minimal level, is not a strategy typically employed by U.S. educators when children exhibit potentially harmful behaviors.

\section{Minimal intervention that maximizes children's learning}

U.S. educators also raised a question regarding what children may learn from educators' minimal intervention. One U.S. teacher, for instance, questioned, "[Mr. Yoshida] just walked up and ...didn't separate them or anything. I found [it] interesting." Mr. Yoshida elaborated why it was not necessary to separate the two boys in this scene, "Hitting with their hands is OK. I would pull them apart if they were hitting using a weapon." An administrator of Sakura Preschool further explained, "It's important that we allow children to 
experience a feeling of 'It hurts!' or feel guilty, 'Oh no, I shouldn't have done it!'” In short, educators at Sakura Preschool do not consider hitting with bare hands as very harmful. Rather, their priority is on creating an opportunity for children to experience physical pain and frustration, from which they can learn that physical fights do not solve any problems. Stopping children's physical fights may take these important learning opportunities away from children.

Similarly, several Japanese educators from the secondary sites described that this scene provides an opportunity for Ken and Takuya to learn from their experience of feeling bad. One teacher described, "I would like to let children experience what it is like when their feelings conflict with each other." Another teacher agreed, "Immediately pulling them apart might be overprotecting them." Japanese educators continued to discuss what they expect children to learn through these consequences of physical fights in the next scene, "non-intervention".

\section{Non-intervention: Keeping children at a distance}

After the minimal intervention in Figure 1, Mr. Yoshida stayed away from children (see Figure 2). This strategy eventually created a situation in which children autonomously solved their own problems (Figure 3).

\section{Children's autonomy in learning}

Mr. Yoshida emphasized the importance of guiding children to learn autonomously. After Ken and Takuya stopped hurting each other, he described, "Educators' intervention, or guidance to help children apologize doesn't mean a lot to them," because that apology is not truly from themselves. This interpretation is supported by not only educators at Sakura Preschool, but also those from the secondary sites. One teacher, for instance, described, "If educators intervene, children are forced to apologize even if they don't want." An administrator further elaborated, "Fighting is an opportunity for children to grow. If educators step in and judge their behaviors, right or wrong, certainly, that can label them as good or bad children. That will definitely affect their relationships negatively."

U.S. educators also showed an understanding of Mr. Yoshida's response, watching the children from a certain distance. One U.S. teacher described, "They came up with their own solution, [although it's] not necessarily the way I would think a problem would be solved." Her colleague also noted, "It seems like the value of plays and being able to work through this and solve it [by themselves]." U.S. educators also acknowledged that the distance between Mr. Yoshida and the two children led another child, Kumi, to step in and mediate the dispute (Figure 3).

\section{Children's learning from conflicts, prioritized over problem solving}

Similar to the use of minimal intervention (Figure 1), by staying away from children (Figure 2), Mr. Yoshida and his colleagues at Sakura Preschool expect that children learn from the experience of feeling bad, which may be more important than solving problems. One teacher, for instance, described, 
In this scene, Kumi didn't have to mediate. Also, it's fine to me if children couldn't solve the problem by themselves. Instead, I wanted them to experience what it is like to have a conflict [with others], for example, thinking and worrying about the other child [with whom they had a conflict], and feeling guilty about hitting and biting.

Mr. Yoshida articulated, "I want them to experience feeling bad after [fighting]. That feeling can make them want to apologize, voluntarily [instead of being forced by adults]."

Likewise, Japanese educators from the secondary sites described the importance of experiencing the consequences of conflicts with others. An administrator, for instance, described, "It's very important for children to experience conflicts, because 'living' in a group means that they will experience [conflicts] and learn that things don't always work as they want. They learn more from experiencing and making sense of conflicts." Another teacher also elaborated,

It's certainly important if children can solve problems by themselves, but what, I think, is more important for their relationship building is to learn from experiencing conflicts and a feeling of uneasy. It takes longer [than when adults intervene more directly], though.

\section{Non-presence of adults: Leaving children in the situation}

When Kumi stepped in to mediate the two boys (Figure 3), Mr. Yoshida determined that no more intervention was necessary, and left the scene (Figure 4). This strategy can be characterized as nonpresence. The difference from non-intervention was that in Figure 2, Mr. Yoshida still stayed close enough to children so that he was able to intervene whenever necessary. In Figure 4, however, his attention was on other children already.

\section{Judging the necessity of further intervention}

Mr. Yoshida explained his action after he determined that there was no longer any immediate risk, "I didn't have to be there, just to watch them arguing. So, I went to play with other children." A Japanese teacher from the secondary site agreed with Mr. Yoshida, and reflected on her own experience, "Once I determine that children can handle [the problem without adults' support], I'll leave them by themselves." By not being present at the site, these educators have created a context in which children are in charge of handling their own conflict. Japanese educators' discussion also underscored that Mr. Yoshida's responses were based on his trust, and confidence in the three children that they were capable of learning from the experience of conflicts. Similarly, in a study by [Author 1] (2014), Japanese early childhood educators created a situation in which children were forced to think and act by themselves by adjusting their location and making their presence out of sight of children.

\section{Meanings of educators' non-presence and no reaction}

Educators further discussed Mr. Yoshida' and other teachers' responses to Ken, Takuya, and Kumi when they walked to the playground after the reconciliation (Figure 4). They indicated that educators in the 
playground did not show any reaction to the three children. U.S. educators, for instance, discussed that such response is not typical in the U.S. One U.S. teacher described, "I was struck by when [children] do go and join the other group... [teachers] are not congratulating or they are giving children no signals." She was impressed that Takuya and Ken made up through Kumi's mediation, and thus considered that Mr. Yoshida should have praised them.

A few Japanese educators from the secondary sites provided the same interpretation. One teacher described, "If I were Mr. Yoshida, I would react to Takuya, Ken, and Kumi [who joined the rest of the children]. I might have told them, 'It was nice that you made up!"' Yet the majority of Japanese educators, both from Sakura Preschool and the secondary sites, supported Mr. Yoshida's no reaction. One teacher from the secondary site described, "Thinking about Takuya, Ken, and Kumi, it is easier for them to join other children [without a feeling of embarrassment], if Mr. Yoshida doesn't show any reaction." A teacher from another site also explained, "Because the teacher shows no reaction, they can join other children as [if nothing has happened], as usual."

U.S. educators' interpretations of this scene, in contrast to the majority of Japanese educators' interpretation, may reflect socialization strategies typically used in the U.S. at home and school, such as the use of praising to guide children in the right direction on how to solve problems (e.g., see Miller \& Cho, 2018). Japanese early childhood educators, however, expressed their preference of leaving the situation to the children. Indeed, Mr. Yoshida expressed his concern, "Praising can impose our values on children, and take away the choices children can make freely." In this particular scene, they considered educators' reactions to children, including praising, as an excessive intervention. Rather, "no reaction" is considered an intervention, which is one of the important features of the Mimamoru approach.

[3] We have excluded references for state regulations to maintain confidentiality of our research sites.

\section{Discussion}

In this study, Japanese and U.S. educators' interpretations of children's physical fight illuminate three major characteristics of the Mimamori approach; that are, temporary minimal intervention, nonintervention, and non-presence. We will discuss, below, any risks and benefits associated with these strategies employed by Mr. Yoshida, which guides us to answer our research questions regarding Japanese educators' use of non-intervention. Generally, a risk, in the context of early childhood education, can be defined as the degree to which injury or disease may occur to children. A benefit, in contrast, is defined as any favorable impact on children (see Cordiano et al., 2019; Gull et al., 2017). Over the past decade, ECEC scholars across cultures have discussed how to balance any risks with benefits associated with children's outdoor play (e.g., Gull et al., 2017). Similarly, we need to consider risks, as well as benefits when using the Mimamoru approach to intervene in children's physical fights.

\section{Why do Japanese early childhood educators tend not to intervene?}


There were two instances in the video clip in which Mr. Yoshida did not intervene. First, Mr. Yoshida's nonintervention by keeping a distance from children who were fighting (Figure 2) can be considered as imposing a risk of physical harm. Children may experience physical pain as a result of hitting and biting each other. Mr. Yoshida, however, observed the situation carefully, for example, "Takuya is not biting seriously", and determined that the benefit for children, such as solving the problem by themselves, outweighed the risk. The expected benefits in this scene also included children's learning by experiencing frustration and a feeling of guilt, or the consequence of conflicts. The opportunity for children to benefit from such experiences might have been taken away, if Mr. Yoshida intervened immediately and eliminated the risk. Still, he was present at the site, with a certain distance from the children, where he was able to intervene if the risk of physical harm escalated.

Next, after Mr. Yoshida determined that there was no further risk of physical harm, he left the site (Figure 4). He understood that children could benefit more from adults' "non-presence". Kumi, for instance, might not have stepped in if Mr. Yoshida stayed there. Children can learn from apologizing to each other with a help of a 5-year-old girl, more than from educators' encouragement.

\section{How and in what contexts, then, do Japanese early childhood educators intervene?}

The only instance Mr. Yoshida directly intervened was when he tried to stop Takuya from biting Ken, by touching Ken's arm without saying anything (Figure 1). He determined that a risk biting could cause overweighed the benefit, and reduced the risk. A closer look at the video, however, revealed that Mr. Yoshida did not immediately intervene. He waited, and observed for a few seconds after Takuya bit Ken's arm to determine whether the risk of physical harm might be greater than any benefit of not intervening. Mr. Yoshida interpreted, in this scene, that the consequence of biting, or bite marks that might remain on Ken's arm could pose a greater risk than the immediate pain Ken was experiencing. Thus, the intervention to eliminate the risk of physical pain, for example, by removing Takuya from the site, might have been understood as an excessive intervention. Such intervention takes away the benefit for children to solve their own problems. Mr. Yoshida only "reduced" the risk, while retaining the benefit by providing a temporary, minimal intervention.

\section{Implications}

The Mimamoru approach has some implications for early childhood education in other contexts, including children's character development (e.g., Ministry of Education, Culture, Sports, Science and Technology, 2017). The Mimamoru approach is considered a pedagogical strategy that maximizes benefits, such as children's acquisition of skills necessary for their development, including social and emotional skills, through the intentional use of non-intervention and non-presence, while reducing potential risks by providing temporary, minimal interventions based on educators' careful observations of children's behaviors. Using the Mimamoru approach, early childhood educators can create a context in which children have autonomy in their actions, for example, older children help younger children solve problems (see also Author 1,2014). Such practice cultivates children's internal motivation to learn new skills. Although it takes time and requires educators' patience to create such contexts, in comparison to 
implementing immediate and more direct interventions, children retain what they have learned when they have ownership of their learning (e.g., Author 3, 2014). Further, as Japanese educators indicated, the Mimamoru approach, which may force children to experience negative consequences of conflicts, is based on educators' trust in children that they can learn from their own experiences (see also Author 3 , 2014, 2020).

This study also suggests that educators' intentional non-presence, non-intervention, and minimal intervention can facilitate children's autonomy to learn, even when intervening in children's physical fights, if educators carefully, and patiently balance the benefits with any risks. Immediate removal of children from physical fights can result in eliminating valuable opportunities for their learning. ECEC around the world, indeed, has emphasized the importance of young children's acquisition, and development of social and emotional skills (e.g., Organization and Economic Co-operation and Development [OECD], 2015), for example, through their experience of handling conflicts, as well as successes (OECD, 2015).

Although each country has different policies for ensuring children's safety at ECEC facilities (e.g., Bayat, 2019; Burke \& Duncan, 2014; Tobin et al., 2009), the Mimamoru approach may suggest new strategies for intervening in children's physical fights in other countries. As U.S. educators in this study indicated, for example, the policies that aim to protect children from any physical harm may not allow educators to wait for children to solve their own problems, particularly, in situations involving physical fights. Yet U.S. participants acknowledged the benefit of Mimamoru approach, such as children's voluntary, and autonomous learning. It may be worth trying out the Mimamoru approach in their classrooms, for example, with parental consent and some modifications to fit with the U.S. educational and policy contexts. Indeed, Vivian Paley, a veteran kindergarten teacher at the University of Chicago Laboratory School eliminated a time-out chair from her classroom, and allowed a child exhibiting aggressive behavior to experience the consequences of his behavior within his peer group. She waited, and encouraged him and his peers to express their thoughts and feelings through role play (Paley, 2004). Although her approach is adult-directed, relative to the Mimamoru approach utilized by Mr. Yoshida, these approaches commonly require educators' patience, rather than punishment, and careful observations of children to minimize potential risks. Using these strategies, educators motivated children to think about how they should behave, and take ownership of their learning.

\section{Limitations}

This study has several limitations that have to be addressed. First, the video clip used in this study to demonstrate the Mimamoru approach is an illustrative example practiced by only one early childhood teacher. His responses to physical fights between the two children may not be considered typical even among Japanese early childhood educators. Future research should examine variations in the use of the Mimamoru approach across Japanese ECEC facilities. Second, we relied on educators' narratives obtained through focus groups. The dynamics within focus groups may have affected narratives provided by participant educators. Moreover, not all participants had opportunities to elaborate their perceptions. Individual interviews with selected educators will allow them to elaborate their 
interpretations, and describe their own experiences with children utilizing the Mimamoru approach. Finally, the video clip used in this study was edited and produced by the NHK as a TV program that has been publicly broadcasted, rather than for the purpose of research. Thus, the video clip does not show the whole scene of the incident involving a physical fight. Mr. Yoshida's non-intervention strategies also may be overemphasized. The edited, short video clip, however, allowed participant educators to reflect on their own practice to fill in the missing scenes and interpret Mr. Yoshida's responses.

\section{Declarations}

\section{Funding}

This study was supported by JSPS (Japan Society for the Promotion of Science) KAKENHI Grant Number JP 23531061.

\section{Conflicts of interest}

We do not have any conflicts of interest.

\section{Availability of data, materials, and codes}

The video clip used in this study is available upon request.

\section{References}

Bamba, S. \& Haight, W. (2011). Child welfare and development: A Japanese case study. Cambridge University Press.

Bayat, M. (2019). Addressing challenging behaviors and mental health issues in early childhood ( $2^{\text {nd }}$ ed.). Routledge.

Burke, R., \& Duncan, J. (2014). Bodies as sites of cultural reflection in early childhood education. Routledge.

Carlson, F.M. (2011). Rough play: One of the most challenging behaviors. Young Children, 66(4), 18-25.

Cordiano, T. S., Lee, A., Wilt, J., Elszasz, A., Damour, L. K., \& Russ, S. W. (2019). Nature-based education and kindergarten readiness: Nature-based and traditional preschoolers are equally prepared for kindergarten. International Journal of early childhood environmental education, 6(3), 18-36.

Doi, T., Lewis, C., Suga, Y., \& Matsuda, Y. (2005). Amae to kyouiku to nihon bunka [Amae, education, and Japanese culture]. PHP Editors Group.

Fujita, M., \& Sano, T. (1988). Children in American and Japanese day-care centers: Ethnography and reflective cross-cultural interviewing. In H.T. Trueba \& C. Delgado-Gaitab (Eds.), School and society: 
Learning content through culture (pp. 73-97). Praeger.

Gull, C., Goldstein, S.L., \& Rosengarten, T. (2017). Benefits and risks of tree climbing on child development and resiliency. International Journal of early childhood environmental education, 5(2), 10-29.

Hayashi, A. (2011). The Japanese hands-off approach to curriculum guidelines for early childhood education as a form of cultural practice. Asia-Pacific Journal of Research in Early Childhood Education, $5(2), 107-123$.

Hayashi, A., Karasawa, M., \& Tobin, J. (2009). The Japanese preschool's pedagogy of feeling: Cultural strategies for supporting young children's emotional development. Ethos, 37(1), 32-49.

Hayashi, A., \& Tobin, J. (2011). The Japanese preschool's pedagogy of peripheral participation. Ethos, 39(2), 139-164.

Hayashi, A., \& Tobin, J. (2012). Reframing a visual ethnography of a Japanese preschool classroom. Visual Anthropology Review, 28(1), 13-31.

Hayashi, A., \& Tobin, J. (2015). Teaching embodied: Cultural practice in Japanese preschools. The University of Chicago Press.

Japan Society of Research on Early Childhood Care and Education (2012) Hoikugaku Kenkyu Rinri Gaidobukku [Code of Ethics for Early Childhood Education Research]. Froebel-Kan

Kubo, Y. (2011). The development of preschool children's conflict resolution with peers: Changes from four-year-olds to six-year-olds. The Bulletin of the Faculty of Sociology Toyo University, 42(2), 41-56

LeVine, R.A., \& White, M.I. (2003). Educational mobilization: The case of Japan. In R. A. LeVine (ed.). Childhood socialization: Comparative studies of parenting, learning and educational change.

Comparative Education Research Centre, The University of Hong Kong

Lewis, C.C. (1995). Educating hearts and minds: Reflections on Japanese preschool and elementary education. New York: Cambridge University Press.

Lincoln, Y.S., \& Guba, E.G. (1985). Naturalistic inquiry. Sage Publications.

Miller, P.J., \& Cho, G.E. (2018). Self-esteem in time and place: How American families imagine, enact, and personalize a cultural idea. Oxford University Press.

Ministry of Education, Culture, Sports, Science and Technology (2017). Youchien kyouiku youryo [National curriculum standards for early childhood education]. Frobel Kan.

Ministry of Education, Culture, Sports, Science and Technology (2018). Youchien kyouiku youryo kaisetsu [Instructional manual for the national curriculum standards for early childhood education]. Frobel Kan. 
Ministry of Education, Culture, Sports, Science and Technology (2019). Youji kyouiku no genjyou [Current status of early childhood education]. Retrieved from https://www.mext.go.jp/content/1421925_08.pdf

NHK (2012, May 18). Naite Waratte [Crying and Smiling]. NHK.

NHK (2021). Kyouiku kenkyu mokuteki deno NHK bangumi no riyou wo o-kangae no kata he [For those who are planning to use our programs for research and education]. Retrieved from https://www.nhk.or.jp/nijiriyou/kyouiku.html\#top

OECD (2015). OECD Skills Studies: Skills for Social Progress - The Power of Social and Emotional Skills. Paris: OECD Publishing. https://doi.org/10.1787/9789264226159-en

Otani, T. (2019). Shitsuteki kenkyu no kangaekata: Kenkyu houhouron kara SCAT ni yoru bunseki made [Paradigm and design of qualitative study: From research methodology to SCAT]. The University of Nagoya Press.

Paley, G.V. (2004). A child's work: The importance of fantasy play. University of Chicago Press.

Peak, L. (1991). Learning to go to school in Japan: The transition from home to preschool life. University of California Press.

Tobin, J. (1989) Visual anthropology and multivocal ethnography: A dialogical approach to Japanese preschool class size. Dialectical Anthropology, 13, 173-187.

Tobin, J., \& Hayashi. A. (2015). Using video for microanalysis of teachers' embodied pedagogical practices. Research in Comparative \& International Education, 10(3), 326-336.

Tobin, J., Hsueh, Y. \& Karasawa, M. (2009). Preschool in three cultures Revisited. The University of Chicago Press.

Tobin, J., Wu, D., \& Davidson, D. (1989). Preschool in three cultures. Yale University Press.

The World Bank (2017). Early childhood development. Retrieved from https://www.worldbank.org/en/topic/earlychildhooddevelopment\#3

U.S. Department of Education (2017). School climate and discipline: Rethinking discipline. Retrieved from https://www2.ed.gov/policy/gen/guid/school-discipline/index.html\#featured

Walsh, D.J. (2004). Frog boy and the American monkey: The body in Japanese early schooling. In L. Bresler (Ed), Knowing bodies, moving minds: Towards embodied teaching and learning (pp. 97-109). Kluwer Academic.

Wang, J., Elicker, J., McMullen, M., \& Mao, S. (2008). Chinese and American preschool teachers' beliefs about early childhood curriculum. Early Child Development and Care, 178(3), 227-249. 


\section{Figures}

This figure has been temporarily replaced with this placeholder.

\section{Figure 1}

Mr. Yoshida, trying to stop Takuya who is biting Ken's arm

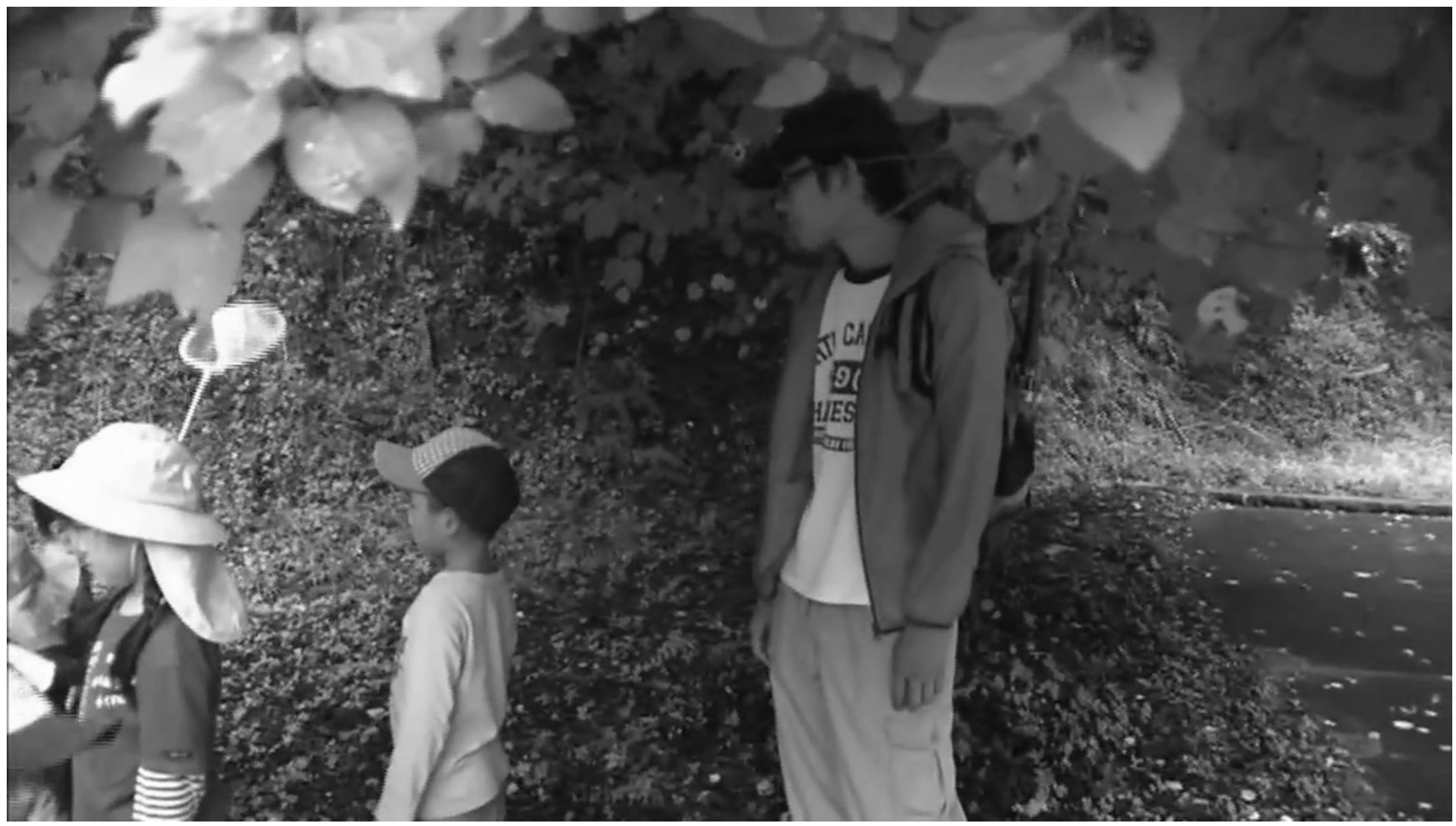

Figure 2

Mr. Yoshida, stepping back from children 
This figure has been temporarily replaced with this placeholder.

\section{Figure 3}

Kumi, mediating the fight between Ken and Takuya

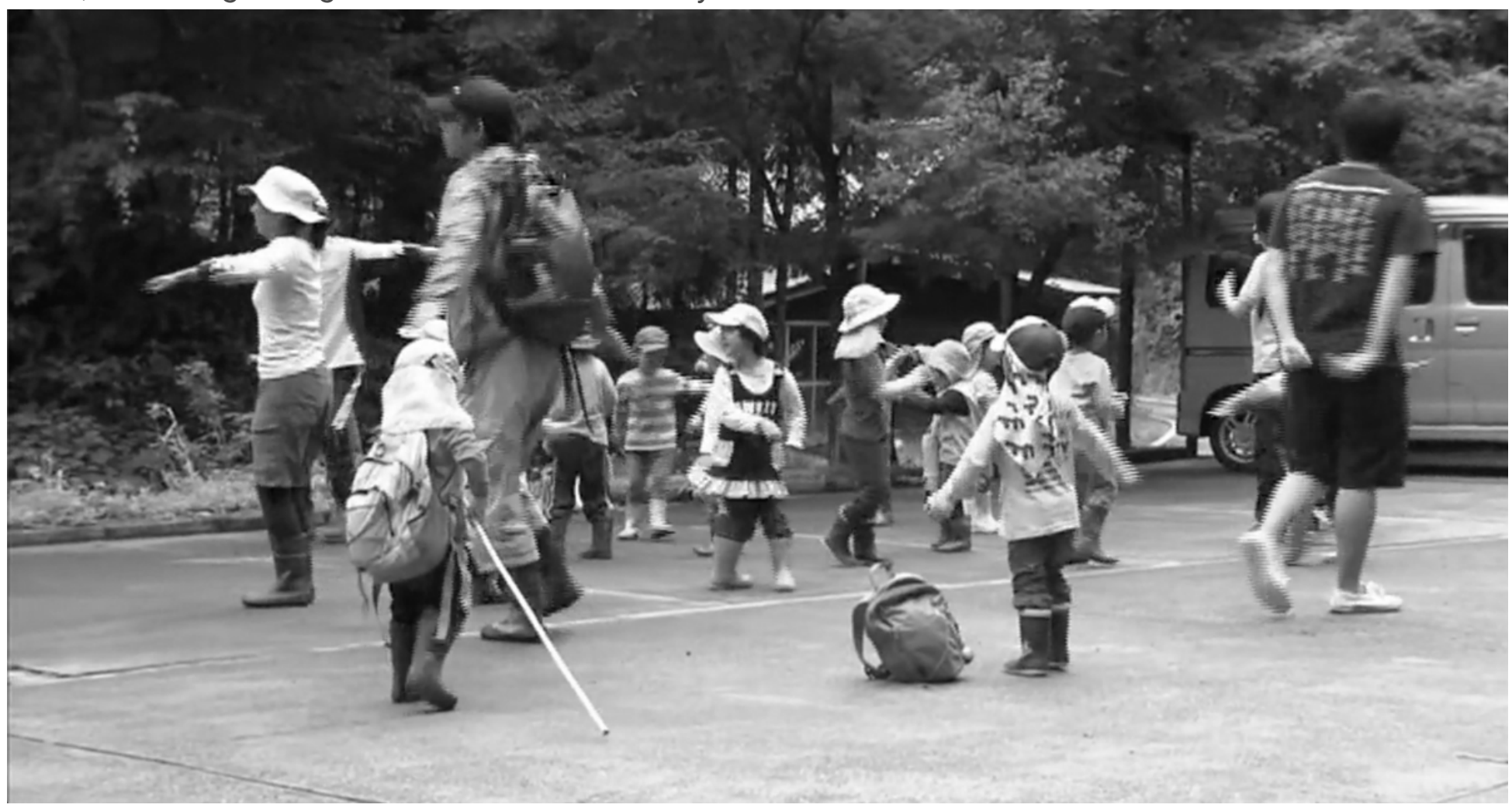

Figure 4 